\title{
An Advanced Well-differentiated Pancreatic Neuroendocrine Carcinoma (NET-G3) Associated with Von Hippel-Lindau Disease
}

\author{
Masami Miki ${ }^{1}$, Ken Kawabe ${ }^{1}$, Hisato Igarashi ${ }^{1}$, Tatsuro Abe ${ }^{2}$, Yoshihiro Ohishi ${ }^{2}$, \\ Risa Hashimoto ${ }^{3}$, Takashi Karashima ${ }^{4}$, Ichiro Yamasaki ${ }^{4}$, Keiji Inoue $^{4}$, \\ Tetsuhide Ito ${ }^{1}$ and Yoshihiro Ogawa ${ }^{1}$
}

\begin{abstract}
:
A 45-year old woman who underwent several surgeries for tumors associated with von Hippel-Lindau disease (VHL) was referred to our hospital due to a pancreatic tumor and liver tumors. She was diagnosed with pancreatic neuroendocrine tumor (NET) with a Ki67 index of $40 \%$ based on the examination of a biopsy specimen of the liver tumors. She was treated with everolimus for 6 months and sunitinib for 6 weeks as first- and second-line therapies. She survived for 13 months. At autopsy the diagnosis of pancreatic neuroendocrine tumor (NET)-G3 was confirmed. We herein report an aggressive clinical course of VHL-related NET G3. The further accumulation of cases is required to reach a consensus on treatment for this disease.
\end{abstract}

Key words: pancreatic neuroendocrine tumors-G3 (NET-G3), well-differentiated neuroendocrine carcinoma (WDNEC), von Hippel-Lindau disease (VHL), WHO G3, everolimus

(Intern Med 57: 2007-2011, 2018)

(DOI: 10.2169/internalmedicine.0416-17)

\section{Introduction}

Von Hippel-Lindau disease (VHL) is a hereditary syndrome with an autosomal dominant feature due to a germline mutation of the $V H L$ tumor suppressor gene residing in chromosome 3p25.5 (1). Patients with VHL are at risk of developing various neoplasms, such as central nervous system (CNS) tumors and renal cell carcinoma. Pancreatic neuroendocrine neoplasms are another manifestation associated with VHL. Approximately $10-17 \%$ of VHL patients will develop PNET (2); however, the rate of advanced cases of VHL-related pancreatic neuroendocrine tumor (PNET) with metastasis is reported to be lower than that in patients with sporadic PNET (3).

According to the WHO 2017 classification (a proliferation-based grading system), PNETs are categorized as NET G1 [mitotic count, $<2$ per 10 high-power fields (HPFs) and/or a Ki67 index of <2\%], NET G2 (mitotic count 2-20 per $10 \mathrm{HPFs}$ and/or a Ki67 index of 3-20\%), NET G3 (well-differentiated type; mitotic count. $>20$ per 10 HPFs and/or a Ki67 index of 20\%) and NEC G3 (poorly differentiated type: mitotic count, $>20$ per $10 \mathrm{HPFs}$ and/or a Ki67 index of 20\%). Among the three grades, G3 tumors, which account for $7.5 \%$ of all PNETs in Japan, generally tend to progress most aggressively (4). Due to their rarity, there is currently no consensus on the therapy for advanced VHL-related PNET, especially on cases corresponding to G3.

We herein report a case of VHL-associated PNET G3 that progressed rapidly in spite of molecular targeted therapies.

\footnotetext{
${ }^{1}$ Department of Medicine and Bioregulatory Science, Graduate School of Medical Sciences, Kyushu University, Japan, ${ }^{2}$ Department of Anatomic Pathology Science, Graduate School of Medical Sciences, Kyushu University, Japan, ${ }^{3}$ Department of Gastroenterology, Saiseikai Fukuoka General Hospital, Japan and ${ }^{4}$ Department of Urology, Kochi Medical School, Kochi University, Japan

Received: October 29, 2017; Accepted: December 12, 2017; Advance Publication by J-STAGE: February 28, 2018

Correspondence to Dr. Ken Kawabe, kkawabe@intmed3.med.kyushu-u.ac.jp
} 

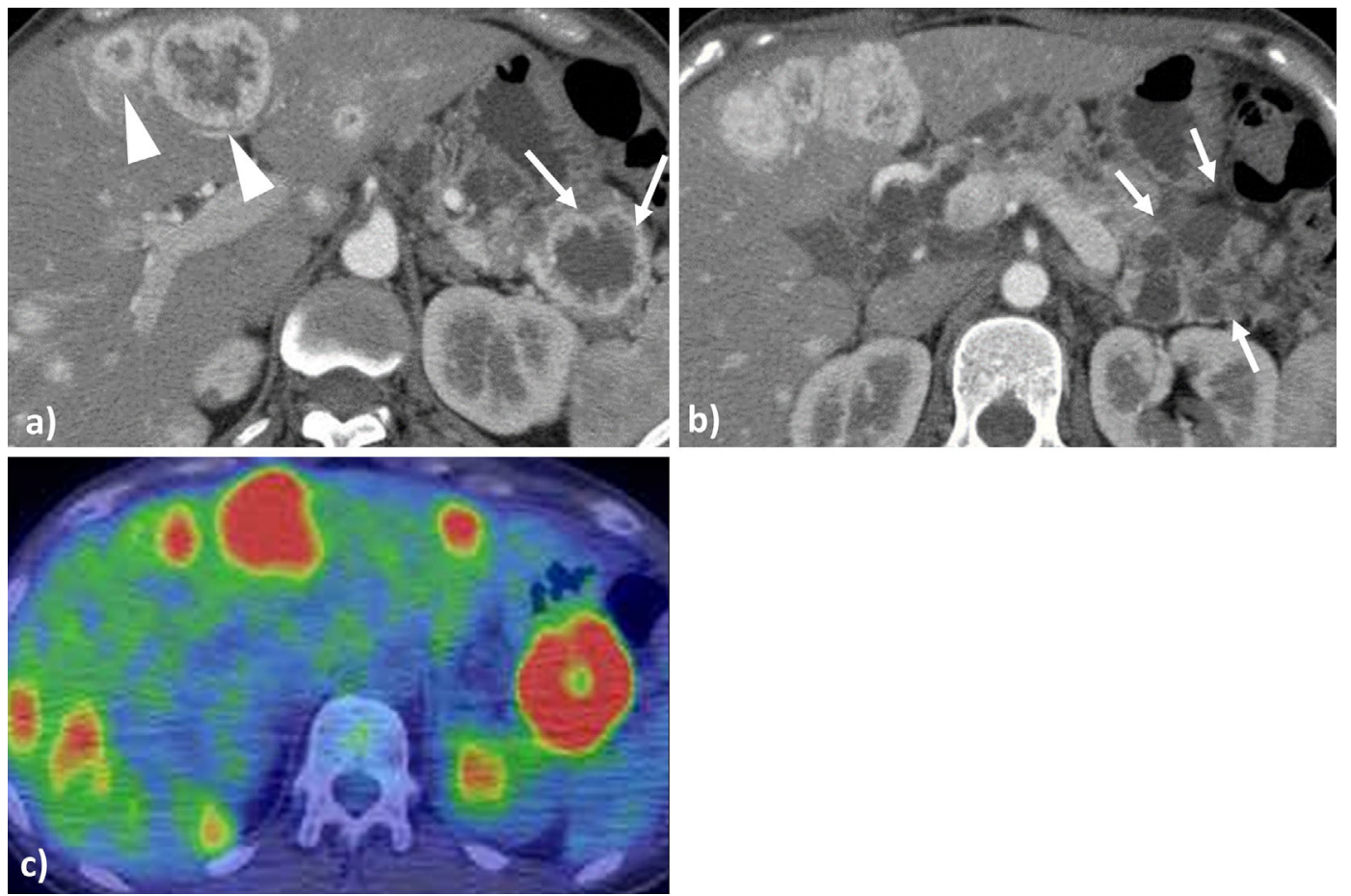

Figure 1. Images on the first admission. a: Computed tomography (CT) showed a hyper-vascular mass with a hypo-vascular lesion in the center of the pancreatic tail (arrow) and liver masses (arrowhead). b: CT also showed multiple pancreatic cysts (arrow), which were compatible with simple cysts or serous neoplasms. c: ${ }^{18} \mathrm{~F}$-fluorodeoxy glucose-positron emission tomography showed the abnormal uptake of the mass lesions.

\section{Case Report}

A 45-year old Japanese woman was referred to our hospital for the management of a pancreatic tumor and multiple liver tumors. At 17 years of age, she underwent surgical resection of a cerebellar hemangioblastoma and was diagnosed with VHL following a genetic examination. Genome sequencing revealed a nonsense mutation c.394C $>\mathrm{T}$ (p.Gln132X) in exon 2. There was no evidence of a family history of VHL. She experienced multiple recurrent cerebellar hemangioblastoma and underwent three additional surgical operations. She also had a history of surgery of retinal hemangioblastoma and adrenalectomy for pheochromocytoma at age of 26 and 30 age years of age, respectively.

She presented no symptoms at admission to our hospital. The initial abdominal physical examination revealed no palpable mass and there was no pronounced tenderness.

Routine blood tests revealed no abnormal findings, with the exception of anemia and mildly elevated alkaline phosphatase (ALP) $412 \mathrm{U} / \mathrm{L}$ and gamma-glutamyl transpeptidase $(\gamma$-GTP)164 U/L levels. Her serum level of chromogranin A $57.8 \mathrm{ng} / \mathrm{mL}$ (normal range, $<47 \mathrm{mg} / \mathrm{mL}$ ) and neuron-specific enolase (NSE) was $15.4 \mathrm{ng} / \mathrm{mL}$ (normal range, $<15.1 \mathrm{mg}$ / $\mathrm{mL}$ ).

Abdominal CT revealed a 4-cm mass with ring enhancement in the pancreatic tail and multiple liver tumors (Fig. 1a), and also showed diffuse pancreatic cystic lesions, which were compatible with simple cysts or neoplasms, as the pancreatic involvement of VHL (Fig. 1b). ${ }^{18} \mathrm{~F}-$ fluorodeoxy glucose positron emission tomography (FDGPET)-CT imaging showed the increased uptake in both the pancreatic and liver tumors with maximal standardized uptake values (SUV-max) of 14.0 and 21.1 respectively (Fig. 1c). The examination of a percutaneous liver tumor biopsy specimen led to a diagnosis of NET G3 (Ki-67 index, 40\%; mitotic count, <2 per $10 \mathrm{HPFs}$ ) according to the 2017 World Health Organization (WHO) classification. Hematoxylin and Eosin (HE) staining of the specimen should features that were more likely well-differentiated than poorlydifferentiated. Immunohistochemically, the tumor cells were positive for synaptophysin, CD56, AE1/AE3, CAM5.2 and negative for chromogranin A.

We suggested the treatment choices to the patient, including systematic chemotherapy using a platinum-based regimen and molecular targeted therapy. She selected the latter using everolimus, a mammalian target of rapamycin (mTOR) inhibitor. Everolimus was started at a daily dose of $10 \mathrm{mg}$ and no severe adverse events were observed. CT image at 3 months after the initiation of everolimus showed no progression of the primary or metastatic tumors. However, a further 3 months later, her liver metastases increased in size. As a second-line therapy, she was given a multi-tyrosine kinase inhibitor sunitinib. Sunitinib as administered at the initial dose of $37.5 \mathrm{mg}$, daily for 2 weeks; the dose was then reduced to $25 \mathrm{mg}$ daily because of adverse events (hy- 

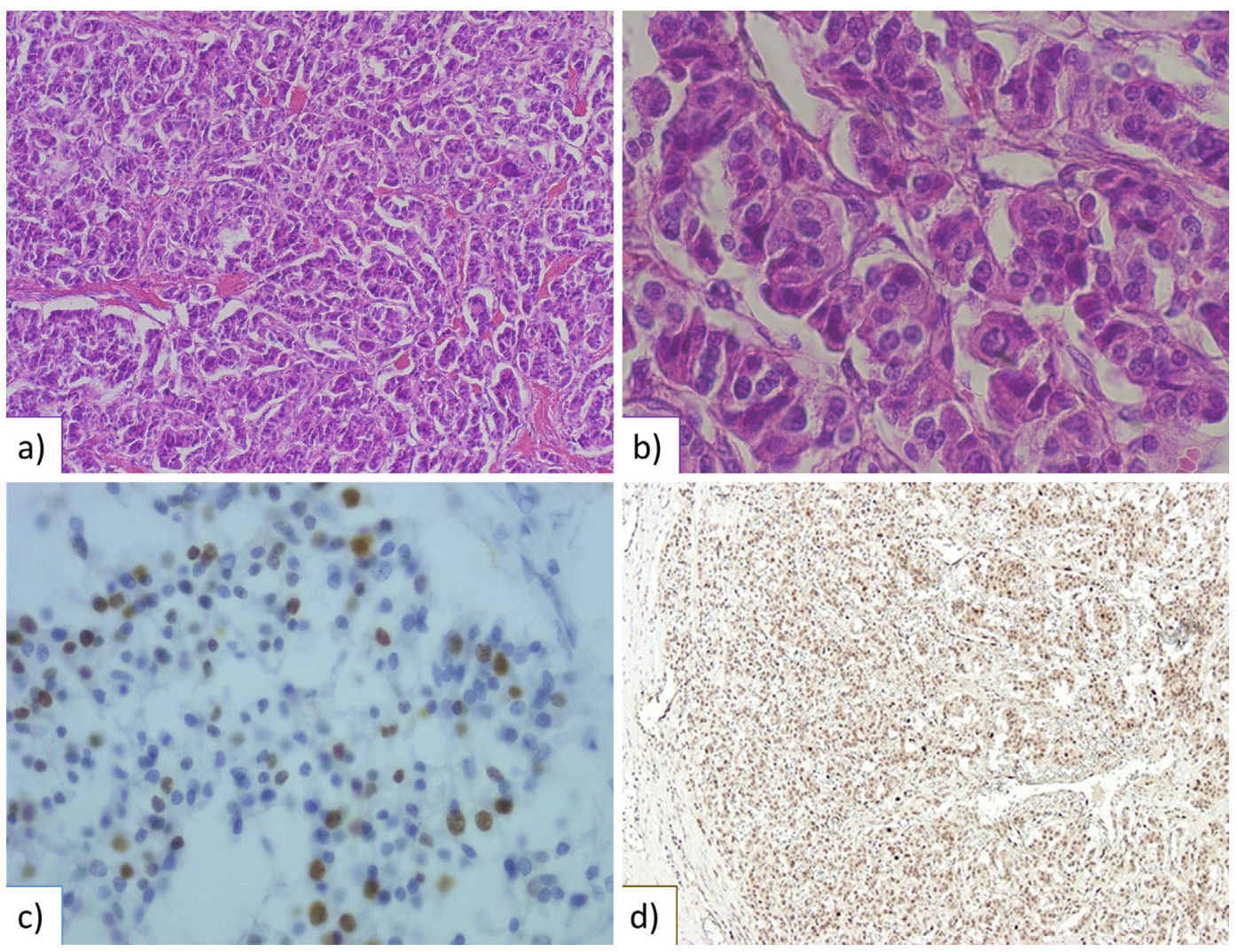

Figure 2. A microscopic examination of the pancreatic tumor (Hematoxylin and Eosin staining) showed nests of small polygonal cells, separated by thin fibrous septae and typical salt and pepper chromatin in each cell (magnification. a: $\times 100, b: \times 400)$. c: Immunohistochemistry showed that the Ki-67 labeling index of the pancreatic tumor was $25 \%$ (MIB-1 staining, magnification 100x). d: The retained expression of $\mathbf{R b}$ in the pancreatic neuroendocrine tumor was indicated by immunohistochemistry (magnification 40x).

pertension and facial edema). A one month after the dose reduction, sunitinib therapy was stopped because the size of liver metastases had significantly increased. After treating the patient with molecular targeted therapies, which showed low effectiveness, we introduced platinum-based chemotherapy as a third-line therapy, based on the consideration that the tumor was composed of poorly-differentiated cells. However, everolimus was eventually re-attempted. Unfortunately, the liver metastases increased in size and number at 3 months after the second administration of everolimus. Although the administration of irinotecan plus cisplatin was initially performed as a next step, the tumor progression led to the gradual deterioration of her general condition. Thus, chemotherapy was discontinued during the first course. She died just 13 months after the initial diagnosis of PNET. An autopsy was performed.

Macro-pathologically, a 5-cm multinodular tumor was located in the pancreatic tail and several serous cystic neoplasms were seen in the pancreas. In addition, multiple brown tumors and white nodules were found scattered in the liver and lungs, respectively. Micro-pathologically (Fig. 2), the Ki-67 index of the primary tumor was $25 \%$ and the number of mitotic cells seen in the tumor was approximately 1 per 10 HPFs. The final diagnosis was NET-G3, be- cause of the low nuclear grade and because the cell morphology of the primary neuroendocrine neoplasm differed from large or small cell carcinoma. Immunohistochemistry (IHC) revealed that the tumor was positive for synaptophysin, chromogranin A and CD56 and negative for gastrin, insulin, glucagon, somatostatin and vasoactive intestinal peptide (VIP). Additionally, IHC revealed Rb immunopositivity. In addition, the findings from the pathological examination of the liver and lung tumors were consistent with metastases of PNET. Furthermore, some necrosis and the disappearance of tumor cells was seen in the liver tumor, which might reflect the influence of molecular targeted therapy.

\section{Discussion}

VHL is hereditary cancer syndrome with an autosomal dominant feature due to a germline mutation of the VHL tumor suppressor gene residing in chromosome 3p25.5 (1). Patients with VHL frequently develop central nervous system (CNS) tumors including cerebellar, brainstem, nerve root, and supratentorial haemangioblastomas. In addition, renal cell carcinomas (RCC), phaeochromocytomas, pancreatic cysts and PNET may develop. Haemangioblastomas of CNS and RCC are considered to be common causes morbidity in 
VHL patients $(5,6)$. However, few patients die due to pancreatic involvement. The mortality rate of patients with pancreatic manifestations is reported to be only $0.5 \%$ (3/ 633) (7). Generally, in comparison to sporadic PNET, VHLassociated PNET is reported to have low-malignant potential (8-10), and it rarely results in death. In Japan, PNET with metastasis was found in only $7.5 \%$ of VHL patients and no PNET-related deaths were reported among patients with VHL-associated PNET (3). The fact that the majority of VHL-associated PNET cases involved G1 or G2 disease (11) may be one of the reasons for the better prognosis of patients with VHL-related PNET. However, in the present case, PNET led to death and the survival time was only 13 months. Blansfield et al. reported that a primary lesion of $>3 \mathrm{~cm}$ in size, a doubling time of $<500$ days and exon 3 mutation of $V H L$ are predictors of malignant potential in VHL-associated PNET (7). In the present case, the tumor was over $3 \mathrm{~cm}$ in size and the tumor doubling time was 208 days before treatment, which indicated aggressive behavior. However, the mutation site was in exon 2 (not exon 3), which did not correspond to the previous report.

A search of the Pubmed database using the search term, "Neuroendocrine carcinoma" and "von Hippel Lindau Disease (VHL)" or "Neuroendocrine neoplasm" and "WHO G3" and "VHL" revealed no reports. To the best of our knowledge, this is the first report on the treatment of advanced VHL-associated PNET G3.

Recently, the concept that PNET with a Ki-67 index of $>20 \%$ includes 2 categories-well-differentiated neuroendocrine carcinoma (WDNEC) and poorly-differentiated neuroendocrine carcinoma ( PDNEC) -was established $(12,13)$. After the WHO 2010 classification, pancreatic NEC was divided into NET G3 and NEC G3 in the WHO 2017 classification (14). In contrast to NEC G3, platinum-based chemotherapy was shown to be ineffective for NET G3 (15). However, it is difficult to distinguish between NET G3 and NEC G3 in some cases (including the present case). Tang et al. (12) reported that IHC to detect $\mathrm{Rb}$ may help to distinguish between these two entities, because the rates of PDNEC and WDNEC in cases that were negative for the expression of $\mathrm{Rb}$ (as assessed by IHC) were $86 \%$ and $0 \%$, respectively. It was also shown that platinumbased chemotherapy is less effective for Rb-positive PNET than it is for Rb-negative PNET (16). The present case was classified as NET G3 based on the cell morphology, and the $\mathrm{Rb}$ immunopositivity of the tumor cells. Thus, a poor response to platinum-based chemotherapy could be predicted in the present case.

Everolimus, a molecular targeted drug was used as a firstline therapy for this patient. The efficacy of everolimus for PNET G1 and G2 was shown in a clinical trial (17). Furthermore, the administration of everolimus was associated with good outcomes in WDNEC. The progression-free survival (PFS) and overall survival were 6 months and 28 months, respectively (18). In our case, first-line therapy with everolimus resulted in PFS for 6 months. Although this indi- cated that everolimus would be effective, the result was worse than the results observed in patients with NET G1 and $\mathrm{G} 2$.

Sunitinib, which was used as the second-line therapy for this case, is a multi-tyrosine kinase inhibitor targeting signals of vascular endothelial growth factor (VEGF) receptor 1,2 and platelet-derived growth factor (PDGF)-beta receptor, which is related to tumor vascularity, metastasis, and proliferation (19). The antitumor efficacy of sunitinib in the treatment of PNET G1/G2 was also shown in a clinical trial (19). According to a genetic analysis (20), tumor angiogenesis factors, including VEGFR1 and 2, are expressed at higher levels in patients with VHL-related PNET than in those with sporadic PNET. To support this, there is a report of a case in which sunitinib therapy resulted in a good prognosis in a patient with VHL-related PNET (19). However, in the present case, the response to sunitinib was ultimately unfavorable.

On the other hand, streptozocin, which was shown to be effective and safe for WDNEC (NET G3) (21), might have been an option in this case. However, it was not on the market in Japan at that time. Greater clinical experience is required to build a consensus on the efficacy of everolimus and sunitinib therapy in cases of VHL-associated NET G3.

We reported an advanced case of VHL-associated PNET, which followed an aggressive clinical course. Further cases of NET G3 with VHL should be accumulated.

The authors state that they have no Conflict of Interest (COI).

\section{References}

1. Lonser RR, Glenn GM, Walther M, et al. Von Hippel-Lindau disease. Lancet 361: 2059-2067, 2003.

2. Kulke MH, Anthony LB, Bushnell DL, de Herder WW, et al. NANETS treatment guidelines: well-differentiated neuroendocrine tumors of the stomach and pancreas. Pancreas 39: 735-752, 2010.

3. Igarashi $\mathrm{H}$, Ito $\mathrm{T}$, Nishimori $\mathrm{I}$, et al. Pancreatic involvement in Japanese patients with von Hippel-Lindau disease: results of a nationwide survey. J Gastroenterol 49: 511-516, 2014.

4. Ito $\mathrm{T}$, Igarashi $\mathrm{H}$, Kazuhiko $\mathrm{K}$, et al. Epidemiological trends of pancreatic and gastrointestinal neuroendocrine tumors in Japan: a nationwide survey analysis. J Gastroenterol 50: 58-64, 2015.

5. Maddock IR, Moran A, Maher ER, et al. A genetic register for von Hippel-Lindau disease. Genet 33: 120-127, 1996.

6. Richard S, Campello C, Taillandier L, Parker F, Resche F. Haemangioblastoma of the central nervous system in von HippelLindau disease. French VHL Study Group. J Intern Med 243: 547553, 1998.

7. Blansfield JA, Choyke L, Morita SY, et al. Clinical, genetic and radiographic analysis of 108 patients with von Hippel-Lindau disease (VHL) manifested by pancreatic neuroendocrine neoplasms (PNETs). Surgery 142: 814-818, 2007.

8. Charlesworth M, Verbeke CS, Falk GA, Walsh M, Smith AM, Morris-Stiff G. Pancreatic lesions in von Hippel-Lindau disease? a systematic review and meta-synthesis of the literature. J Gastrointest Surg 16: 1422-1428, 2012.

9. Erlic Z, Ploeckinger U, Cascon A, et al. Systematic comparison of sporadic and syndromic pancreatic islet cell tumors. Endocr Relat Cancer 17: 875-883, 2010. 
10. Yamasaki I, Nishimori I, Ashida S, Kohsaki T, Onishi S, Shuin T. Clinical characteristics of pancreatic neuroendocrine tumors in Japanese patients with von Hippel-Lindau disease. Pancreas 33: 382-385, 2006.

11. Ali T, Kandil D, Piperdi B. Long-term disease control with sunitinib in a patient with metastatic pancreatic neuroendocrine tumor (NET) associated with Von Hippel-Lindau syndrome (VHL) To. Pancreas 41: 492-493, 2012.

12. Tang LH, Basturk O, Sue JJ, Klimstra DS. A practical approach to the classification of WHO grade 3 (G3) well-differentiated neuroendocrine tumor (WD-NET) and poorly differentiated neuroendocrine carcinoma (PD-NEC) of the pancreas. Am J Surg Pathol 40: 1192-1202, 2016.

13. Vélayoudom-Céphise FL, Duvillard P, Foucan L, et al. Are G3 ENETS neuroendocrine neoplasms heterogeneous? Endocr Relat Cancer 20: 649-657, 2013.

14. Lloyd RV, Osamura RY, Klöppel G, Rosai J. Who Classification of Tumours of Endocrine Organs (World Health Organization Classification of Tumours). 4th ed. IARC, Lyon, 2017.

15. Sorbye $\mathrm{H}$, Welin S, Langer SW, et al. Predictive and prognostic factors for treatment and survival in 305 patients with advanced gastrointestinal neuroendocrine carcinoma (WHO G3): The NORDIC NEC study. Ann Oncol 24: 152-160, 2013.

16. Hijioka S, Hosoda W, Mizuno N, et al. Does the WHO 2010 clas- sification of pancreatic neuroendocrine neoplasms accurately characterize pancreatic neuroendocrine carcinomas? J Gastroenterol 50: 564-572, 2015.

17. Yao JC, Shah MH, Ito T, et al. Everolimus for advanced pancreatic neuroendocrine tumors for the RAD001 in advanced neuroendocrine tumors, third trial (RADIANT-3) study group. N Engl J Med 364: 514-523, 2011.

18. Panzuto F, Rinzivillo M, Spada F, et al. Everolimus in pancreatic neuroendocrine carcinomas G3. Pancreas 46: 302-305, 2017.

19. Raymond E, Dahan L, Raoul JL, et al. Sunitinib malate for the treatment of pancreatic neuroendocrine tumors. N Engl J Med 364: 501-513, 2011.

20. Speisky D, Duces A, Bièche I, et al. Molecular profiling of pancreatic neuroendocrine tumors in sporadic and von Hippel-Lindau patients. Clin Cancer Res 18: 2838-2849, 2012.

21. Aoki T, Kokudo N, Komoto I, et al. Streptozocin chemotherapy for advanced/metastatic well-differentiated neuroendocrine tumors: an analysis of a multi-center survey in Japan. J Gastroenterol 50: 769-775, 2015.

The Internal Medicine is an Open Access article distributed under the Creative Commons Attribution-NonCommercial-NoDerivatives 4.0 International License. To view the details of this license, please visit (https://creativecommons.org/licenses/ by-nc-nd/4.0/).

(C) 2018 The Japanese Society of Internal Medicine Intern Med 57: 2007-2011, 2018 